\title{
Development and Characterization of a Theranostic Micelles System using TPGS with Docetaxel and Coumarin-6: A Dual Polymeric Nanocarrier System
}

\author{
${ }^{1}$ L.H. Ching, ${ }^{2,3}$ S. Mahmood , ${ }^{2}$ R.Edros, ${ }^{2,3 *}$ R.V.Kutty \\ ${ }^{1}$ Faculty of Chemical \& Natural Resources Engineering, \\ ${ }^{2}$ Faculty of Engineering and Technology \\ ${ }^{3}$ Center of Excellence for Advanced Research in Fluid Flow, University Malaysia Pahang, 26300, Kuantan, \\ Pahang, Malaysia \\ Universiti Malaysia Pahang, 26300 Gambang, Pahang, MALAYSIA. \\ *Email : vrajaletchumy@ump.edu.my
}

\begin{abstract}
Theranostic micelles and polymeric nanocarrier-based drug delivery system are well known techniques that involve a diagnostic agent in polymeric micelles for a combination of therapy by using a codelivery approach which can help in detection of a cancer cell in an early stage, increase killing effect and suppress multi-drug resistance (MDS) for better therapeutic effectiveness. The aim of this study is to develop a dual modality micellar system using D- $\alpha$-tocopheryl polyethylene glycol 1000 succinate (TPGS) as a nanocarrier for co-delivery of docetaxel as a model chemotherapeutic drug and coumarin-6 as a model fluorescence imaging agent for simultaneous cancer imaging and therapy in an early stage. The theranostic micelles were prepared by a solvent casting method and characterized by their particle size, drug loading, drug encapsulation efficiency (EE) and in-vitro drug release profile. These dual modality micellar system TPDC6 micelles were successfully developed with average particle size of $79.59 \pm 0.57 \mathrm{~nm}$ in diameter and drug loading up to $15.46 \pm 1.02 \%$ (EE of $78.99 \pm 1.26 \%$ ) and $9.83 \pm 0.76 \%$ (EE of $36.20 \pm 0.89 \%$ ) for docetaxel and coumarin- 6 respectively. Besides, the in-vitro drug release profile of the micelles revealed a desired sustained and controlled drug release manner for both docetaxel $(21.62 \pm 0.36 \%)$ and coumarin- 6 $(10.70 \pm 0.46 \%)$. In conclusion, the micelles size obtained is in the favourable range for passive targeting through enhanced permeability and retention (EPR) effect and the drug loading and encapsulation efficiency attained are adequate for therapy and diagnosis purposes on cancer cells. This dual modality system is taking great advantages for tumour imaging and inhibition of tumour growth which is very important for early cancer detection.
\end{abstract}

Indexed Terms- Nanomedicine; Polymeric micelle; Docetaxel; Coumarin-6, Biomaterial, Chemotherapeutic

\section{I.INTRODUCTION}

Cancer is commonly characterized by uncontrolled cell growth caused by up-regulation of oncogenes or down-regulation of tumour suppressor genes and angiogenesis [1]. It is also one the leading causes of death overall the world. Molecular subtypes of breast cancer include luminal A, luminal B, basal-like breast cancer, triple negative breast cancer (TNBC) and epidermal growth factor receptor 2 HER2-positive breast cancer [2]. Breast cancer is classified based on tumour markers of progesterone receptor (PR), estrogen receptor (ER) and HER2 [3]. However, TNBC lack of these three receptor proteins ( $\left.\mathrm{PR}^{-} / \mathrm{ER}^{-} / \mathrm{HER} 2^{-}\right)$. The incidence of TNBC cases in Malaysia is approximately $17 \%$, a rate similar to that found in Western studies [4]. Treatment options 
currently available for TNBC are limited to standard cytotoxicity chemotherapy such as paclitaxel due to the lack of therapeutic target required to effectively deliver drugs to the tumour with a minimal side effect on healthy cells and multidrug resistance (MDR) exhibited by a cancer tumour that prevents delivery of therapeutic agents into tumours [5,6]. However, nanomedicine offers effective solutions to overcome the problems encountered by conventional chemotherapy. Nanomedicines are able to deliver therapeutic agents into the targeted cells via receptor-mediated endocytosis (RME). Further, nanomedicines are also capable of conquering MDR by providing protection to therapeutic agents and prevent elimination of P-glycoproteins (P-gp) [7]. Issues with conventional chemotherapy such as drug resistance, formulation and pharmacokinetics (controlled drug release) may be solved with the assist of nanomedicine.

Patients with TNBC are usually detected at the late stages, have an aggressive tumour type due to the high rate of local recurrences and systematic metastases and a poorer prognosis compared to patients with non-TNBC [8]. In addition, the mean time to local and distant recurrence was shorter in patients with TNBC than other cancers which were 2.8 versus 4.2 years and 2.6 versus 5.0 years respectively [9]. These patients were significantly more likely to have visceral metastases, which might contribute to their poorer prognosis. Hence, there is a clear demand to examine nanotheranostics for sensitive diagnosis at its earliest stage and can effectively use in the treatment of TNBC a tumour by the therapeutic agent.

The aim of this study is to develop and characterize a novel theranostic micelle system for cancer treatment in addition to study the drug release profile of the micellar system. The scopes of study are to synthesize the dual-modality system of docetaxel-coumarin-6-loaded vitamin E TPGS micelles (TPDC6) and optimize it to achieve desired particle size, drug loading and precise drug release in $\mathrm{pH}$ 7.4. Docetaxel is chosen as the model anticancer agent or therapeutic agent whereas coumarin- 6 is chosen as the diagnostics or imaging agent and both are encapsulated by TPGS which serve as their nanocarriers into TNBC cells. Docetaxel leads to a mitotic arrest in the G2/M phase of the cell cycle by binding to microtubules and causes cell death [7]. Coumarin-6 with fluorescence property is able to act as a fluorescent probe for applications such as biomarker and bioimaging to visualize morphological details and monitor biomolecule in living systems [10]. Vitamin E consisting of a lipophilic alkyl tail and a hydrophilic polar head was used to synthesize the polymeric micelle which above its critical micelle concentration (CMC) of $0.02 \mathrm{wt} \%$. The codelivery of both chemotherapeutic agent and diagnosis agent in a dual drug micelle system form a theranostic nanoparticle that can be used in the diagnosis and therapy of breast cancer in an early stage which can control local recurrences and prevent distant metastases [11].

\subsection{Materials}

Docetaxel (anhydrous, 99.56\% purity), Coumarin-6 and Vitamin E TPGS (D- $\alpha$-tocopheryl polyethylene glycol 1000 succinate, $\left.\mathrm{C}_{33} \mathrm{O}_{5} \mathrm{H}_{54}\left(\mathrm{CH}_{2} \mathrm{CH}_{2} \mathrm{O}\right)_{23}\right)$ were obtained from the National University of Singapore (NUS). Chloroform (stabilised with 0.6-1.0\% ethanol) was purchased from R\&M Chemicals. Dichloromethane (DCM) and acetonitrile were all purchased from Fisher Scientific. Tween-80 was provided by Firma Chempur Company. Methanol was obtained from Fisher Scientific Corporation. Phosphate buffered saline (PBS, pH7.4) was prepared in the laboratory from sodium chloride $(\mathrm{NaCl})$, potassium chloride $(\mathrm{KCl})$, disodium phosphate $\left(\mathrm{Na}_{2} \mathrm{HPO}_{4}\right)$ and potassium dihydrogen phosphate $\left(\mathrm{KH}_{2} \mathrm{PO}_{4}\right)$. All solvents used in this research study were high-performance liquid chromatography (HPLC) grade. All chemicals were used without further purification. Ultrapure water was prepared by a Milli-Q Plus System (Millipore Corporation). Amicon ultra-15 centrifugal filter units (molecular weight cut-off (MWCO): 100K) were purchased from Merck Millipore. 


\subsection{Preparation of docetaxel-loaded TPGS micelle (TPD), coumarin-6 loaded TPGS micelle (TPC6) and TPDC6 micelle}

TPD and TPC6 micelles were prepared by solvent casting method. Briefly, docetaxel or coumarin-6 (2 mg) and $100 \mathrm{mg}$ of TPGS were dissolved in $5 \mathrm{~mL}$ of chloroform solution and evaporated using rotary vacuum evaporator at $20^{\circ} \mathrm{C}-30^{\circ} \mathrm{C}$ until a thin film of drug-dispersed TPGS was formed. The thin film was then suspended in $10 \mathrm{~mL}$ of PBS buffer solution ( $\mathrm{pH} 7.4)$ and incubated in an orbital water bath shaker at $37^{\circ} \mathrm{C}$ under constant agitation for $1.5 \mathrm{~h}$, followed by 10 min of sonication. The resultant mixture was filtered through a $0.22-\mu \mathrm{m}$ filter and followed by centrifugal filter units with MWCO $100 \mathrm{kDa}$ (Amicon ultra-15 ml) to separate the excess nonincorporated drug precipitate from the suspension. The resultant suspension was then freezed at $80^{\circ} \mathrm{C}$ and followed by further dried in a freeze-dryer for overnight for $12 \mathrm{hrs}$. The same procedure was used for the synthesis of TPDC6 micelle with the replacement of $2.0 \mathrm{mg}$ of docetaxel and 0.2 $\mathrm{mg}$ of coumarin-6 (10:1 weight ratio) and $100.0 \mathrm{mg}$ of TPGS in $10 \mathrm{~mL}$ suspension.

\subsection{Particle size and size distribution of micelles}

The particle Size and size distribution of the TPD, TPC6 and TPDC6 micelles were measured by photon correlation spectroscopy (PCS) using Zetasizer (Nano ZS, Malvern Instrument Ltd, Malvern, UK). The micelle samples were first prepared by diluting the nanocarriers suspension with ultrapure water to a count rate of 300-500 kilo counts per second (kcps) and then sonicated for $5 \mathrm{~min}$ before measurement to ensure that the micelles were well dispersed [12]. All the measurements were recorded in triplicates $(n \pm S . D)$.

\subsection{Drug loading and encapsulation efficiency}

The amount of docetaxel encapsulated in TPDC6 and TPD micelles was measured by highperformance liquid chromatography (HPLC, Agilent LC1100, Agilent, Tokyo, Japan) in a reversephase column (Eclipse XDB-C18, 4.6 x $250 \mathrm{~mm}, 5 \mu \mathrm{m}$ ). Briefly, $1 \mathrm{~mL}$ of micelles was freeze-dried and dissolved in $1 \mathrm{~mL}$ of DCM to break the polymer matrix. After evaporating the DCM overnight, $0.5 \mathrm{~mL}$ of mobile phase (50:50 \% v/v acetonitrile and ultrapure water) was added to dissolve the extracted drugs. After centrifugation at 13,000 rpm for $5 \mathrm{~min}$, a supernatant of the suspension was collected and the solution was then filtered through a $0.45 \mu \mathrm{m}$ PVDF syringe filter before being transferred into an HPLC vial for HPLC analysis. The drug encapsulation efficiency was defined as the ratio between the amount of docetaxel encapsulated in the micelles and that added in the micelles preparation process. The drug loading was calculated as the weight of the drug encapsulated in the micelles divided by the total weight of the micelles $[13,14]$. The drug loading and encapsulation efficiency of coumarin-6 encapsulated in TPDC6 and TPC6 micelles were determined by ultraviolet/visible (UV-VIS) spectrophotometer (Hitachi, U-1800) at $430 \mathrm{~nm} .1 \mathrm{~mL}$ of methanol was added into a designated amount of freeze-dried micelle sample that was then vortexed until all freeze-dried micelle dissolved completely. The concentration of coumarin- 6 in the micelle was detected by the UV-VIS spectrophotometer and the total amount of encapsulated coumarin- 6 was calculated. All the measurements were recorded in triplicates $(n \pm S . D)$.

$$
\begin{gathered}
\text { Drug Loading }=\frac{\text { Weight of drug encapsulated }}{\text { Total weight of micelles }} \times 100 \% \quad \text { Equ. (1) } \\
\text { Drug Entrappment efficinecy }=\frac{\text { mass of drug in nanoparticles }}{\text { mass of drug used in formulation }} \times 100 \%
\end{gathered}
$$




\subsection{In vitro drug release profile}

The centrifugal ultrafiltration technique was used to study the in vitro drug release profile of docetaxel and coumarin- 6 from micelles. One $\mathrm{mL}$ solutions of TPD, TPC6 and TPDC6 micelles were respectively added into the ultrafiltration membrane of centrifugal filter units with MWCO 10 $\mathrm{kDa}$ and immersed in $30 \mathrm{~mL}$ of PBS $(0.1 \mathrm{M}$, neutral $\mathrm{pH} 7.4)$ containing $0.1 \%(\mathrm{v} / \mathrm{v})$ Tween-80 in the filtrate collection cup of centrifugal filter unit to simulate the sink condition. The entire system was kept at $37^{\circ} \mathrm{C}$ with gentle and constant shaking. At designated time intervals i.e. every $24^{\text {th }} \mathrm{hr}$, the incubation buffer was collected $1 \mathrm{ml}$ and replaced by a fresh incubation buffer. The collected incubation buffer, containing the released drug, was then freeze-dried and dissolved in DCM. The samples were filtered through $0.45 \mathrm{~mm}$ syringe filter before transferred into HPLC vial. The amount of docetaxel released was determined by HPLC method whereas the amount of coumarin- 6 released was determined by UV-VIS spectrophotometer as described in the protocols of drug loading and encapsulation determination (section 2.4). The drug release profiles were calculated accordingly $[12,14]$. All the measurement was recorded in triplicates $(n \pm S . D)$.

\section{RESULT AND DISCUSSION}

\subsection{Micelle particle size analysis}

The particle size of micelles measured by the dynamic light scattering (DLS) are shown in Figure 2, from which it can found that the mean micellar size (average) of the TPD, TPC6 and TPDC6 were $21.36 \pm 0.17 \mathrm{~nm}, 69.06 \pm 0.89 \mathrm{~nm}$ and $79.59 \pm 0.57 \mathrm{~nm}$ respectively, which were in a very favourable range of size for drug delivery in cancer treatment. It was revealed that micellar size typically in the range of $10 \mathrm{~nm}$ to $100 \mathrm{~nm}$ are appropriate for drug delivery in cancer diagnosis and therapy due to the reason of micelles in such size enhances EPR effect to a solid tumour $[14,15]$. The EPR phenomenon is based on the nanometer size range of micelles, leaky vasculature and impaired lymphatic drainage characteristics of the neoplastic tissues, which allowed the small size micelles (less than $100 \mathrm{~nm}$ ) to participate in the extravasation through the fenestrations in tumour vessels and accumulate in the neoplastic tissues [16, 17]. According to Taurin et al. hydrodynamic diameter of more than $7 \mathrm{~nm}$ was able to escape from renal filtration and urinary excretion, leading to exhibit prolonged circulatory half-life in the blood and permit the accumulation of the nanocarriers within the neoplastic tissues. Therefore, TPD, TPC6 and TPDC6 micelles developed in this research (figure.1) could be excellent delivery tools in cancer treatment due to the micelles size of less than $100 \mathrm{~nm}$ [18].

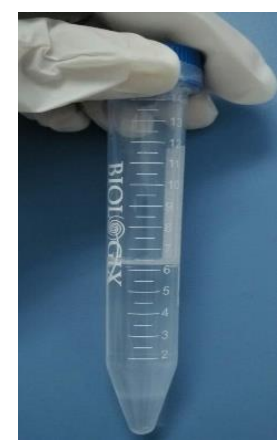

(a)

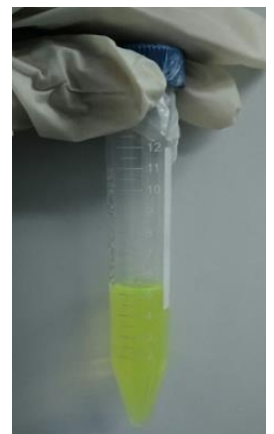

(b)

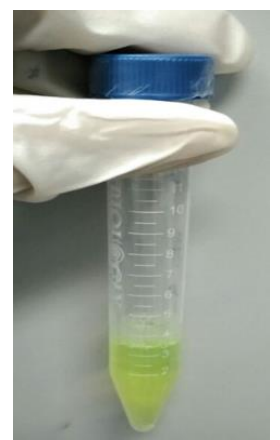

(c)

Figure 1: The produced nanocarriers (a) TPD (b) TPC6 (c) TPDC6 

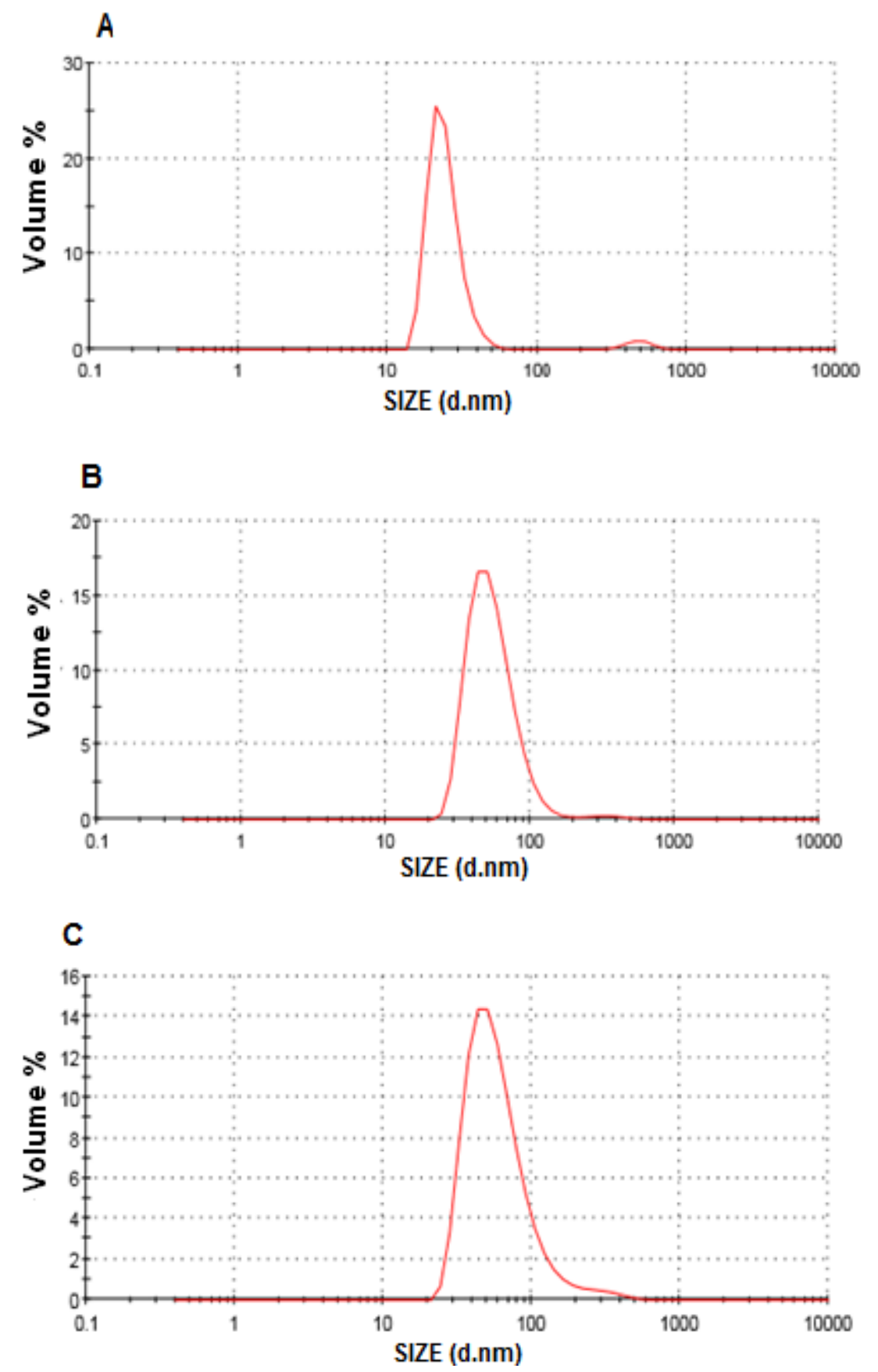

Figure 2: Average particle size of the micelles. (A) TPD (B) TPC6 and (C) TPDC6

\subsection{Drug loading and encapsulation efficiency}

Drug load is determined as the weight of the drug $(\mu \mathrm{g})$ per $\mathrm{mg}$ of the drug-loaded micelles. The results are shown in Table 1 and Table 2. The docetaxel load for TPD and TPDC6 micelles at $16.28 \pm 1.34 \%$ and $15.46 \pm 1.02 \%$ respectively, while the drug loading of coumarin- 6 for TPC6 and TPDC6 micelles are $10.60 \pm 0.89 \%$ and $9.83 \pm 0.73 \%$ respectively. The drug encapsulation efficiency of TPD micelles was $83.02 \pm 1.97 \%$ whereas the dye encapsulation efficiency of TPC6 micelles was $54.05 \pm 1.31 \%$. The data are in close agreement with the research carried out by Muthu and group for similar systems. Similarly, the drug encapsulation efficiency and dye encapsulation 
efficiency of TPDC6 micelles were $78.99 \pm 1.26 \%$ and $36.20 \pm 0.89 \%$ for docetaxel and coumarin- 6 respectively [19].

The drug loading of coumarin-6 for both TPC6 micelles and TPDC6 micelles were lower compared to docetaxel loading, yet, it was high enough for in vivo tracking of nanocarriers and imaging purpose [20]. The possibility for lower drug loading might be due to leakage of coumarin6 and docetaxel during the incubation step. Furthermore, the relatively lower drug loading and encapsulation efficiency of coumarin-6 for TPDC6 micelles could be caused by the difference in hydrophobicity between docetaxel and coumarin-6. According to the study done by Huang et al, coumarin- 6 being more hydrophilic than docetaxel [21], however, Mi and group claimed that drug solubilization was more effective for the most hydrophobic drug than for more hydrophilic ones, hence, it caused lower drug loading and encapsulation for coumarin-6 in TPDC6 micelles [22]. Moreover, the higher amount of docetaxel $(2 \mathrm{mg})$ compared to coumarin-6 $(0.2 \mathrm{mg})$ in the micelles preparation process might be one of the reason. The weight ratio of 10:1 and drug load of $15.46 \pm 1.02 \%: 9.83 \pm 0.76 \%$ for docetaxel and coumarin- 6 respectively were adequate for the therapy purpose by docetaxel and imaging purpose by coumarin- 6 .

Table 1: Drug loading and encapsulation efficiency of docetaxel in TPD and TPDC6 micelles

\begin{tabular}{|l|c|c|}
\hline \multicolumn{1}{|c|}{ Micelles } & Drug load \% & Encapsulation efficiency (\%) \\
\hline TPD & $16.28 \pm 1.34$ & $83.02 \pm 1.97$ \\
\hline TPDC6 & $15.46 \pm 1.02$ & $78.99 \pm 1.26$ \\
\hline
\end{tabular}

Table 2: Drug loading and encapsulation efficiency of coumarin-6 in TPC6 and TPDC6 micelles

\begin{tabular}{|l|c|c|}
\hline \multicolumn{1}{|c|}{ Micelles } & Drug load (\%) & Encapsulation efficiency (\%) \\
\hline TPC6 & $10.60 \pm 0.89$ & $54.05 \pm 1.31$ \\
\hline TPDC6 & $9.83 \pm 0.73$ & $36.20 \pm 0.89$ \\
\hline
\end{tabular}

\subsection{In vitro drug release profile}

In order to facilitate passive targeting to the tumour site, the drug should be released in a controlled manner and obtained sustained circulation to attain sustained therapeutic efficacy. In vitro release study was carried out in PBS pH 7.4 with $0.1 \%$ v/v Tween-80 and immediately replaced by the fresh buffer for simulating the sink condition. Figure 3 and 4 show the in vitro drug release profiles of docetaxel and coumarin-6 respectively, in TPD, TPC6 and TPDC6 micelles for 240 hours. It can be seen from the figures that the drug release profile of the three micelle systems follows a biphasic pattern with an initial burst release followed by controlled release. The initial burst of docetaxel release was $55.25 \pm 1.88 \%$ and $21.62 \pm 0.36 \%$ in the first 50 hours, for the TPD and TPDC6 micelles respectively. Whereas, the initial burst of coumarin- 6 was $16.30 \pm 0.53 \%$ and $10.70 \pm 0.46 \%$ in the first 48 hours, for the TPC6 and TPDC6 micelles respectively. The burst release could be due to the drug poorly encapsulated in the polymeric core and located under the periphery of the nanocarriers while the sustained release was attributed to the diffusion of the wellentrapped drug in the core of nanocarriers [23]. 
After 10 days, the accumulative docetaxel drug release from TPD micelles achieved $64.99 \pm 2.12 \%$, which was significantly higher than that from TPDC6 micelles with only $38.88 \pm 1.81 \%$ drug release. This might be due to the diffusivity of drug which depends on its solubility [24]. As discussed previously, coumarin-6 is more hydrophilic than docetaxel and this might have affected the diffusivity and drug release of docetaxel in TPDC6 micelles. Besides, Reza et al revealed that the mechanisms of drug release were changed with the content in the polymer could be one of the reasons for this phenomenon [25]. Whereas, no significant differences were observed for the accumulative coumarin- 6 drug release from TPC6 and TPDC6 micelles after 10 days, which were $21.63 \pm 0.21 \%$ and $16.86 \pm 0.96 \%$ respectively. The fluorescence probe coumarin- 6 encapsulated within the nanocarriers was acting as tracers in vivo or in vitro due to their relatively low leak from nanocarriers and good sensitivity. The very low release of coumarin- 6 from micelles indicated that coumarin- 6 could be adequate fluorescence probes for micelles behaviour in vivo, and the fluorescence signals detected in the cells or tissue are well represented the distribution of the micelles instead of from release of coumarin-6 from the micelles into the medium [26].

All three micelle systems showed a sustained and controlled drug release, which implies that the drugs can be released slowly and kept for a long period both in vivo and in vitro. This leads to avoiding the repeated administrations and eventually less drug accumulation and toxic effects in the body [ 27, 28].

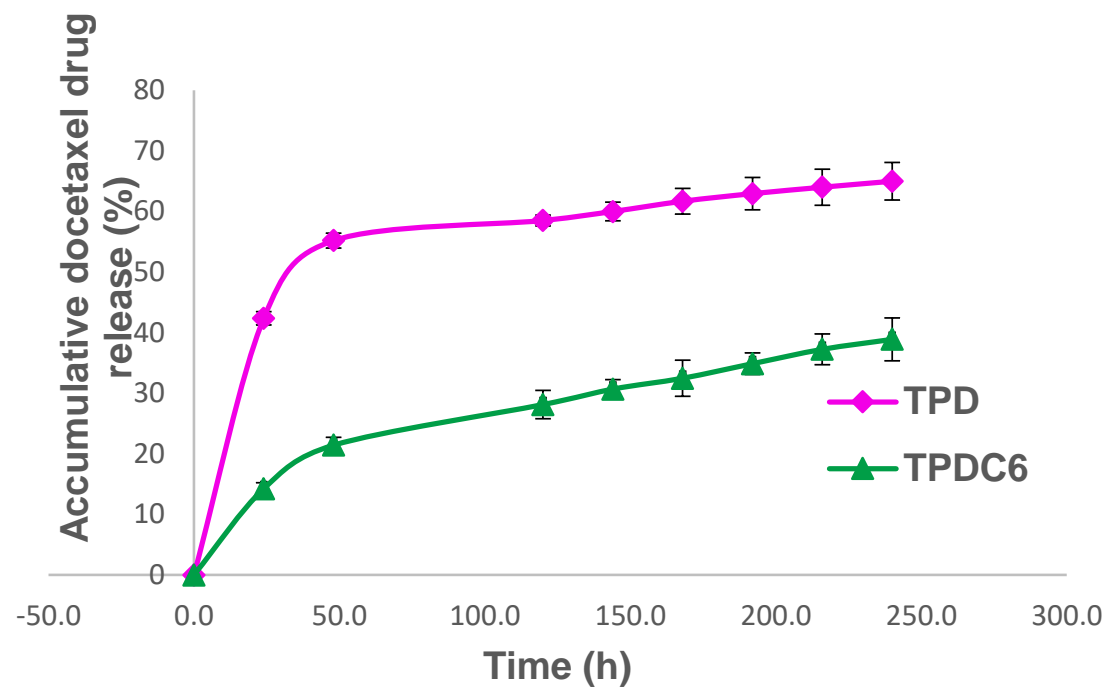

Figure 3: In vitro accumulative docetaxel drug release of TPD and TPDC6 micelles at pH7.4 


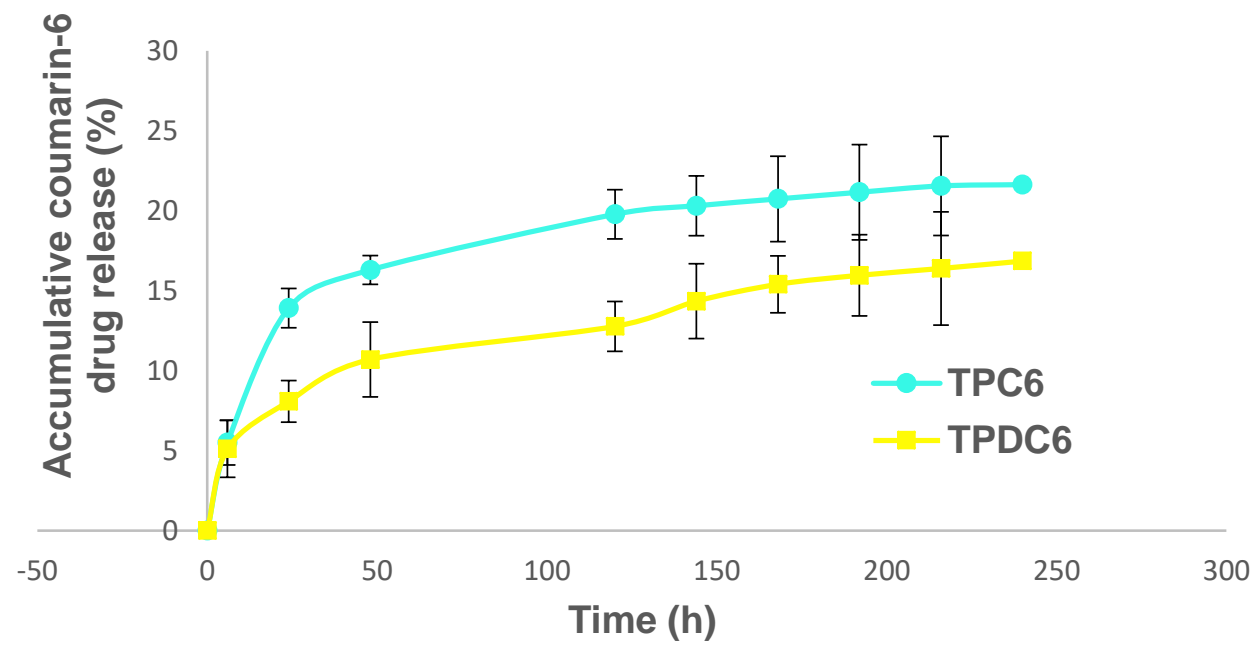

Figure 4: In vitro accumulative coumarin-6 drug release of TPC6 and TPDC6 micelles at pH7.4

\section{CONCLUSION}

In summary, a dual modality system was successfully developed using TPGS micelles for the sustainable and controlled delivery of docetaxel and coumarin-6 into the cancer cells. This research proved that the solvent casting method is considered suitable for the preparation of micelles with co-delivery of therapeutic and diagnostic agents. The DLS and particle size analyser showed that the TPDC6 micelles have an average size of $79.59 \pm 0.57 \mathrm{~nm}$ in diameter. Drug loading up to $15.46 \pm 1.02 \%$ (encapsulation efficiency of $78.99 \pm 1.26 \%$ ) and $9.83 \pm 0.76 \%$ (encapsulation efficiency of $36.20 \pm 0.89 \%$ ) for docetaxel and coumarin- 6 respectively. These results are adequate for the purposes of therapy and diagnosis on cancer cells by docetaxel and coumarin-6 respectively. In vitro, drug release profiles demonstrated a desired sustained and controlled drug release pattern of the micelles which have advantages of less frequent drug administration, maximum utilization of drug and reduction in the side effect of the drug in the body.

There are few recommendations that should be considered for further study and investigation in order to improve the results from this research. From this study, the drug loading and encapsulation efficiency of the dual-modality micelles (TPDC6 micelles) is not in the most excellent condition, hence, it is recommended to optimise the drug loading by varying the weight ratio of docetaxel, coumarin- 6 and TPGS or change a different preparation method to develop the micelles in order to produce a smaller size micelles with higher drug loading and encapsulation efficiency which would be very beneficial in drug delivery. Besides, the drug release profile of TPDC6 micelles in this study showed that the docetaxel drug release was slower and lower compared to TPD micelles. Therefore, further investigation on shape and surface morphology on the micelles could be conducted in order to understand the drug release mechanisms of TPDC6 micelles.

\section{ACKNOWLEDGMENT}

This work was financially supported by a research Grant (Project No. RDU150379) from Universiti Malaysia Pahang (www.ump.edu.my), Malaysia, for which the authors are very grateful. 


\section{REFERENCES}

[1] Siegel, R. L., Miller, K. D., \& Jemal, A. (2015). Cancer statistics, CA Cancer J Clin 2015. 65, 5-29.

[2] Nounou, M. I., ElAmrawy, F., Ahmed, N., Abdelraouf, K., Goda, S., \& Syed-Sha-Qhattal, H. (2015). Breast cancer: conventional diagnosis and treatment modalities and recent patents and technologies. Breast Cancer : Basic and Clinical Research, 9(Suppl 2), 17-34.

[3] Parise, C. A., \& Caggiano, V. (2014). Breast cancer survival defined by the ER/PR/HER2 subtypes and a surrogate classification according to tumor grade and immunohistochemical biomarkers. Journal of cancer epidemiology, 2014.

[4] Dean, S. J., \& Rhodes, A. (2014). Triple negative breast cancer: the role of metabolic pathways. Malays J Pathol, 36(3), 155-162.

[5] Pal, S. K., Childs, B. H., \& Pegram, M. (2012). Triple negative breast cancer: unmet medical needs. 125(3), 627-636.

[6] O’Reilly, E. A., Gubbins, L., Sharma, S., Tully, R., Guang, M. H. Z., Weiner-Gorzel, K., McCann, A. (2015). The fate of chemoresistance in triple negative breast cancer (TNBC). BBA Clinical, 3, 257-275.

[7] Kutty, R. V. (2015). Targeted delivery of Vitamin E TPGS based nanomedicine for treatment of triple negative breast cancer. Thesis submitted for the Degree of Doctor of Philosophy. National University Singapore, Singapore.

[8] Cao, Z., Lee, G., Wang, Y. A., Sajja, H. K., Wang, L., Long, R., ... Yang, L. (2010). Abstract 5482: Theranostic nanoparticles for targeted therapy of triple negative breast cancer and for monitoring therapeutic response by MRI. 70(8 Suppl).

[9] Dent, R., Trudeau, M., Pritchard, K. I., Hanna, W. M., Kahn, H. K., Sawka, C. A., ... Narod, S. A. (2007). Triple-negative breast cancer: clinical features and patterns of recurrence. Clinical Cancer Research, 13(15), 4429-4434.

[10] Mahmood, S., Taher, M., \& Mandal, U. K. (2014). Experimental design and optimization of raloxifene hydrochloride loaded nanotransfersomes for transdermal application. International Journal of Nanomedicine, 9, 4331-4346.

[11] Li, Y., Liu, Q., Li, W., Zhang, T., Li, H., Li, R., ... \& Zhang, Z. (2016). Design and validation of PEG-derivatized vitamin E copolymer for drug delivery into breast cancer. Bioconjugate chemistry, 27(8), 1889-1899.

[12] Zheng, T., Bott, S., \& Huo, Q. (2016). Techniques for accurate sizing of gold nanoparticles using dynamic light scattering with particular application to chemical and biological sensing based on aggregate formation. ACS applied materials \& interfaces, 8(33), 21585-21594.

[13] Muthu, M. S., Kutty, R. V., Luo, Z., Xie, J., \& Feng, S. S. (2015). Theranostic vitamin E TPGS micelles of transferrin conjugation for targeted co-delivery of docetaxel and ultra-bright gold nanoclusters. Biomaterials, 39, 234-248.

[14] Kutty, R. V., Tay, C. Y., Lim, C. S., Feng, S. S., \& Leong, D. T. (2015). Anti-migratory and increased cytotoxic effects of novel dual drug-loaded complex hybrid micelles in triple negative breast cancer cells. Nano Research, 8(8), 2533-2547.

15] Muthu, M. S., Leong, D. T., Mei, L., \& Feng, S. S. (2014). Nanotheranostics - application and further development of nanomedicine strategies for advanced theranostics. Theranostics, 4(6), 660-677.

[16] Bazak, R., Houri, M., El Achy, S., Hussein, W., \& Refaat, T. (2014). Passive targeting of nanoparticles to cancer: A comprehensive review of the literature. Molecular and clinical oncology, 2(6), 904-908.

[17] Steichen, S. D., Caldorera-Moore, M., \& Peppas, N. A. (2013). A review of current nanoparticle and targeting moieties for the delivery of cancer therapeutics. European Journal of Pharmaceutical Sciences, 48(3), 416-427.

[18] Taurin, S., Nehoff, H., \& Greish, K. (2012). Anticancer nanomedicine and tumor vascular permeability; where is the missing link?. Journal of controlled release, 164(3), 265-275. 
[19] Muthu, M. S., Kulkarni, S. A., Liu, Y., \& Feng, S. S. (2012). Development of docetaxel-loaded vitamin E TPGS micelles: formulation optimization, effects on brain cancer cells and biodistribution in rats. Nanomedicine, 7(3), 353-364.

[20] Tan, G. R., Feng, S. S., \& Leong, D. T. (2014). The reduction of anti-cancer drug antagonism by the spatial protection of drugs with PLA-TPGS nanoparticles. Biomaterials, 35(9), 3044-3051.

[21] Huang, J., Zhang, H., Yu, Y., Chen, Y., Wang, D., Zhang, G., ... \& Lu, Y. (2014). Biodegradable self-assembled nanoparticles of poly (d, 1-lactide-coglycolide)/hyaluronic acid block copolymers for target delivery of docetaxel to breast cancer. Biomaterials, 35(1), 550-566.

[22] Mi, Y., Zhao, J., \& Feng, S. S. (2012). Vitamin E TPGS prodrug micelles for hydrophilic drug delivery with neuroprotective effects. International journal of pharmaceutics, 438(1), 98-106.

[23]Tang, S. C., \& Lo, I. M. (2013). Magnetic nanoparticles: essential factors for sustainable environmental applications. Water research, 47(8), 2613-2632.

[24] Buckley, S. T., Frank, K. J., Fricker, G., \& Brandl, M. (2013). Biopharmaceutical classification of poorly soluble drugs with respect to "enabling formulations". European Journal of Pharmaceutical Sciences, 50(1), 8-16.

[25] Reza, M. S., Quadir, M. A., \& Haider, S. S. (2003). Comparative evaluation of plastic, hydrophobic and hydrophilic polymers as matrices for controlled-release drug delivery. J Pharm Pharm Sci, 6(2), 282-91.

[26] Kulkarni, S. A., \& Feng, S. S. (2011). Effects of surface modification on delivery efficiency of biodegradable nanoparticles across the blood-brain barrier. Nanomedicine, 6(2), 377-394.

[27] Zheng, C., Zheng, M., Gong, P., Deng, J., Yi, H., Zhang, P., ... \& Cai, L. (2013). Polypeptide cationic micelles mediated co-delivery of docetaxel and siRNA for synergistic tumor therapy. Biomaterials, 34(13), 3431-3438.

[28] Xiao, H., Song, H., Yang, Q., Cai, H., Qi, R., Yan, L., ... \& Jing, X. (2012). A prodrug strategy to deliver cisplatin (IV) and paclitaxel in nanomicelles to improve efficacy and tolerance. Biomaterials, 33(27), 6507-6519. 